\title{
Multivariate analysis in pediatric brain tumor
}

\begin{abstract}
Brain tumors in children are life-threatening and deserve more research to improve patient care. In recentyears, multivariate analysis has been increasingly used in tumor classification (segmentation) and survival (outcome) assessment in childhood brain tumors. This paper reviewed the studies that applied multivariate analysis to tumor classification (segmentation) and survival (outcome) assessment in pediatric brain tumors. Large variations in the tumor classification results were observed in the studies of tumor classification (even in similar patient populations). Moderate error rate in the multivariate survival analysis model was also observed, which could lead to inaccurate survival estimates and misidentification of prognostic factors. To address these problems, this paper analyzed the data processing chains in these multivariate analyses in detail. It seems that optimizing and standardizing these data processing chains may improve tumor classification and survival analysis, reduce variations and errors in classification results and survival estimates. As multivariate analytic approaches, data processing technologies and imaging techniques advance in the Big Data era of the 21 st century, it is anticipated that the challenges in complex imaging data processing in tumor classification will be overcome and complex data processing will be revolutionized. This will make accurate automatic tumor classification/ segmentation (for each tumor type and grade) possible to early detect and treat tumors, guide treatment planning, monitor tumor progression and treatment effects, together with advanced accurate survival assessment to guide life-saving rescue and recovery planning, revolutionize patient care, and truly benefit children with brain tumors.
\end{abstract}

Keywords: multivariate analysis, pediatric brain tumor, tumor classification, survival assessment
Volume 2 Issue 6 - 2017

\author{
Jing Zhang \\ Department of Pediatric Surgery, University of Texas Health \\ Science Center at Houston, USA
}

\begin{abstract}
Correspondence: Jing Zhang, Department of Pediatric
Surgery, University of Texas Health Science Center at Houston, Houston, Texas, USA, Tel 8324193193,
\end{abstract}

Email jzhang0000@gmail.com

Received: April 21, 2017| Published: April 26, 2017
Abbreviations: DWI, diffusion-weighted imaging; MRS, $\mathrm{mr}$ spectroscopy; PWI, perfusion-weighted imaging; SVM, support vector machine; LDA, linear discriminant analysis; $\mathrm{kNN}$, k-nearest neighbour; ANN, artificial neural networks; KLD, kullback-leibler divergence; $\mathrm{mBm}$, multi-fractional brownian motion; EM, expectation maximization; PNET, primitive neuroectodermal tumor; PTPSA, piecewise-triangular-prism-surface-area; MD, mean diffusivity; FA, fractional anisotropy; PCA, principal component analysis; mRMR, maximum-relevance minimum redundancy; DNET, dysembryoplastic neuroepithelial Tumor; DNT, dysembryoplastic neuroepithelial tumor; ADC, apparent diffusion coefficient; rADC, ADC tumor-to-normal-brain ratios; ROC, receiver operating characteristic; AUC, area under the roc curve; ATCT, apparent transient coefficient in tumor; OS, overall survival; PFS, progression-free survival; GTR, gross total resection; RT, radiation therapy

\section{Introduction}

Brain and central nervous system tumors are the $2^{\text {nd }}$ most common tumors in children. ${ }^{1}$ Compared with brain tumors in adults, the histological types of childhood brain tumors have a larger variety such as medulloblastomas, pilocytic astrocytomas and ependymomas, which increases the difficulty in tumor diagnosis and differentiation. Since brain tumors in children are life-threatening, they deserve more research to improve patient care.

MRI (including T1, T2, FLAIR and contrast-enhanced MRI) is the most important imaging technique for the visualization and assessment of pediatric brain tumors. However, conventional MRI technique is often inadequate in grading tumors or identifying the aggressive region of the tumor. Therefore, advanced MRI techniques (modalities) such as DWI (Diffusion Weighted Imaging), MRS (MR
Spectroscopy) and PWI (Perfusion Weighted Imaging) are needed to help with tumor assessment, and are increasingly used in brain tumor diagnosis. DWI is based on water diffusion and the apparent diffusion coefficient (ADC) map that DWI generates provides information on the conditions of different brain tissues. ${ }^{2}$ MRS provides information on the specific molecules' concentration in the brain tissue which reflects the biochemical characteristics in that brain region. PWI provides information on vascular perfusion (blood flow in capillaries and larger vessels) of the brain. These imaging techniques are useful in classifying (typing) and grading brain tumors. ${ }^{3}$

Multivariate analysis is a statistical approach that assesses multiple variables simultaneously, which may be more advantageous than univariate analysis in characterizing the associations between data variables (e.g., variables associated with outcomes), classifying data into different categories (e.g., tumor types and grades) and generating new diagnostic tests. Multivariate analysis includes a number of analytic methods such as multivariate regression, principal components analysis (PCA), independent components analysis (ICA) and cluster analysis. Machine learning (statistical learning) is a group of multivariate analytic methods often used in data classification, pattern recognition and data mining. ${ }^{2}$ Machine learning can be either supervised or unsupervised. In supervised learning, the classifications of data samples in the training set (to train the classification model or classifier) are known; while in unsupervised learning, the classifications of data samples in the training set are unknown. Examples of supervised learning methods include linear discriminate analysis (LDA), support vector machine (SVM), artificial neural networks (ANN) and random forests. Examples of unsupervised learning include cluster analysis and Hebbian learning neural networks. 
Since tumor classification and patient survival assessment often have much larger number of data variables than the sample sizes, they are called high-dimensional problems. Multivariate analysis is suitable for such problems. Imaging analytic studies of brain tumors in adults have been developed for decades, but imaging research of brain tumors in children is relatively young. ${ }^{2}$ In recentyears, multivariate analysis has been increasingly used in tumor classification (or segmentation) and survival (or outcome) assessment in childhood brain tumors. This paper reviewed the studies that applied multivariate analysis to tumor classification (segmentation) and survival (outcome) assessment in pediatric brain tumors.

\section{Methods}

Pubmed search was performed with keywords "multivariate
MRI pediatric brain tumor", "machine learning imaging brain tumor childhood" or "multivariate pediatric brain tumor". The search yielded 41 articles, and articles were excluded if their subjects were not pediatric or the statistical methods used were not multivariate or the article was published before year 2000 .

\section{Results}

27 articles were selected in this review. Among them, 13 articles reported multivariate analysis methods applied to pediatric brain tumor classification (or segmentation). ${ }^{3-15}$ Table 1 provides a summary of these papers. The rest of the articles reported multivariate analysis (using Cox model) applied to survival (or outcome) evaluation in pediatric brain tumors, ${ }^{16-29}$ which are summarized in Table 2.

Table I Summary of multivariate analysis approaches applied to pediatric brain tumor classification/segmentation

\begin{tabular}{|c|c|c|c|c|}
\hline Paper & $\mathbf{N}$ & Methods & Main Findings & Other Findings \\
\hline Schneider et al. ${ }^{4}$ & $\begin{array}{l}\text { I } 7 \text { pts with posterior } \\
\text { fossa tumors ( } 7 \\
\text { medulloblastoma, } 4 \\
\text { infiltrating glioma, } 2 \\
\text { ependymoma, } 4 \text { pilocytic } \\
\text { astrocytoma) }\end{array}$ & $\begin{array}{l}\text { MRI, DWI, and MRS } \\
\text { acquired and imaging } \\
\text { parameters computed; linear } \\
\text { discriminant analysis used as } \\
\text { classifier }\end{array}$ & $\begin{array}{l}\text { Combined ADC and } \\
\text { metabolite ratio features } \\
\text { (using water as an } \\
\text { internal standard) could } \\
\text { discriminate between } \\
\text { the four tumor groups } \\
\text { (positive predictive } \\
\text { rate }=1 \text { ), likelihood below } \\
1 \times 10(-9)\end{array}$ & $\begin{array}{l}\text { Metabolite ratio or ADC features } \\
\text { alone could not discriminate the } \\
\text { tumor groups }\end{array}$ \\
\hline Reynolds et al.., & $\begin{array}{l}46 \text { pts (I } 6 \text { astrocytoma } \\
\text { grade I and II, I } 3 \\
\text { medulloblastoma, } \\
3 \text { ependymoma , } 3 \\
\text { germinoma, } 3 \text { PNET, } 2 \\
\text { astrocytoma grade III and } \\
\text { IV, and } 6 \text { rare tumors) }\end{array}$ & $\begin{array}{l}\text { I } 333 \text { cases in WMRCTR } \\
\text { database were used to } \\
\text { produce probabilities of } \\
\text { brain tumor class and } \\
\text { construct classifiers } \\
\text { (Bayesian belief networks); } \\
\text { incorporate MRS information } \\
\text { into the classifier }\end{array}$ & $\begin{array}{l}\text { Overall misclassification } \\
\text { rate: } 32-37 \% ; 4.8-25.6 \% \\
\text { for individual tumor class }\end{array}$ & $\begin{array}{l}\text { Using the network to generate } \\
\text { prior probabilities improves } \\
\text { classification accuracy when } \\
\text { compared with class prevalence- } \\
\text { based prior probabilities }\end{array}$ \\
\hline Wels et al.., & 6 pts with tumors & $\begin{array}{l}\text { Graph cut top-down } \\
\text { segmentation method } \\
\text { (with max-flow/min-cut } \\
\text { optimization) for tumor } \\
\text { segmentation; probabilistic } \\
\text { boosting trees as classifier }\end{array}$ & $\begin{array}{l}\text { Jaccard } \\
\text { coefficient }=0.78 \pm 0.17\end{array}$ & $\begin{array}{l}\text { Automatic tumor segmentation; } \\
\text { results comparable to those in } \\
\text { adult patients; takes less time than } \\
\text { manual segmentation }\end{array}$ \\
\hline Davies et al.., & $\begin{array}{l}35 \text { pts with } \\
\text { cerebellar tumors ( } 18 \\
\text { medulloblastomas, } 12 \\
\text { pilocytic astrocytomas } \\
\text { and } 5 \text { ependymomas) }\end{array}$ & $\begin{array}{l}\text { Short-TE MRS acquired } \\
\text { and metabolite profiles } \\
\text { computed and verified; linear } \\
\text { discriminant analysis used } \\
\text { for variable selection and } \\
\text { classification }\end{array}$ & $\begin{array}{l}\text { Misclassification rate: } 5.3 \% \\
\text { for glial-cell (astrocytoma } \\
+ \text { ependymoma) } \\
\text { vs. non-glial-cell } \\
\text { (medulloblastoma) } \\
\text { tumors; } 6.9 \% \text { for } \\
\text { astrocytoma vs. } \\
\text { medulloblastoma; } 7.1 \% \\
\text { for astrocytoma vs. } \\
\text { medulloblastoma vs. } \\
\text { ependymoma }\end{array}$ & $\begin{array}{l}\text { Medulloblastomas characterised } \\
\text { by high taurine, phosphocholine } \\
\text { and glutamate and low glutamine; } \\
\text { Astrocytomas distinguished by } \\
\text { low creatine and high NAA; } \\
\text { Ependymomas differentiated } \\
\text { by high myo-inositol and } \\
\text { glycerophosphocholine }\end{array}$ \\
\hline
\end{tabular}


Table Copntinued.

$\begin{array}{llll}\text { Paper N Methods } \quad \text { Main Findings } & \text { Other Findings }\end{array}$

$\begin{array}{ll}\text { Ahmed et al., } & \text { fossa tumors ( } 5 \\ & \text { medulloblastomas, } 5 \\ & \text { astrocytomas) }\end{array}$

Iftekharuddin et al.,

10 pts with posterior fossa tumors ( 5 medulloblastomas, 5 astrocytomas)
Training set: 7 pts (28 scans) with optic pathway gliomas (OPG); Test set: 5 pts with OPG (25 scans)
Shape, intensity and (fractal dimension and multifractional Brownian motionbased) texture features extracted from TI,T2, and FLAIR images; Feature selection (PCA, boosting, $\mathrm{KLD}$, entropy); Feature fusion (EM)
$\mathrm{mBm}$-based texture features produced the best tumor segmentation accuracy for TI and FLAIR images and for TI-T2-FLAIR fused images (I00\%), and intensity features best for T2 images; KLD measure for feature ranking and selection, and EM algorithm for feature fusion and tumor segmentation generated the best results; average Jaccard Index for tumor segmentation: $\sim 0.6$

Shape, intensity and (fractal dimension and multifractional Brownian motionbased) texture features extracted from TI,T2, and

FLAIR images; Improved AdaBoost classifier

Tumor segmentation based on prior tumor location tissue characteristics, and intensity information; classification of tumor into internal components; automatic volume measurements for tumor follow-up evaluation

78 pts $(29$

medulloblastomas,

Vicente et al.," II ependymomas; 38 pilocytic astrocytomas)
MRS acquired and metabolite concentrations computed; linear discriminant analysis and resampling used as classification
True positive rate $=100 \%$ False positive rate $=25 \%$
Integrated KLD-EM framework is the best for tumor segmentation compared with other approaches (such as bottom up top down and graph cut)

Combined features improved classification accuracy
A mean surface distance error of $0.73 \mathrm{~mm}$ and a mean volume overlap difference of $30.6 \%$, with 25 min less segmentation time, compared with manual segmentation by radiologists

Discriminate the three tumor types, Balanced Accuracy Rate $(B A R)=0.98$

ADC histogram features generated the best classification accuracy $(95.8 \%$ of medulloblastomas, $96.9 \%$ of pilocytic astrocytomas, and 94.3\% of ependymomas; ADC textural features produced the best tumor-subtype classification accuracy (89\% of medulloblastoma subtypes); combined features: $91.4 \%$ of the joint posterior fossa tumors
Automatic tumor segmentation; Can monitor tumor growth; Can be used to segment and classify other tumors such as intraventricular and posterior fossa tumors

For other tumor types (glial or primitive neuroectodermal): $B A R=1.00$

Shape and textural features did not improve classification accuracy of ADC histogram feature-based classification performance single-feature SVM combined into multi-feature classifiers pilocytic astrocytomas,
and 7 ependymomas)
Shape, histogram, and Rodriguez
Gutierrez et al., ${ }^{12}$ 
Table Continued..

\begin{tabular}{|c|c|c|c|c|}
\hline Paper & $\mathbf{N}$ & Methods & Main Findings & Other Findings \\
\hline $\begin{array}{l}\text { Tantisatirapong } \\
\text { et al.,., }\end{array}$ & $\begin{array}{l}74 \text { pts ( } 25 \\
\text { medulloblastomas, } 34 \\
\text { pilocytic astrocytomas, } \\
\text { and } 15 \text { ependymomas) }\end{array}$ & $\begin{array}{l}\text { Data from CCLG database; } \\
\text { Texture features extracted } \\
\text { from TI,T2, FLAIR,ADC, MD } \\
\text { and FA images; PCA, mRMR } \\
\text { and feedforward methods } \\
\text { used for feature selection; } \\
\text { SVM classifier }\end{array}$ & $\begin{array}{l}\text { Classification } \\
\text { accuracy }=69 \%\end{array}$ & $\begin{array}{l}\text { Texture features extracted from } \\
\text { diffusion MR images are stronger } \\
\text { for tumor classification than those } \\
\text { from MRI }\end{array}$ \\
\hline Fetit et al.., ${ }^{14}$ & $\begin{array}{l}48 \text { pts ( } 2 \text { I } \\
\text { medulloblastoma, } 20 \\
\text { pilocytic astrocytoma, } 7 \\
\text { ependymoma) }\end{array}$ & $\begin{array}{l}\text { 2D and 3D texture features } \\
\text { (grey-level co-occurrence } \\
\text { matrix, etc.) extracted from } \\
\text { MRI (TI,T2); Entropy-MDL } \\
\text { discretization for feature } \\
\text { selection; } 6 \text { classification } \\
\text { methods used: Naïve Bayes, } \\
\text { k-nearest neighbour (kNN), } \\
\text { Classification tree, SVM, } \\
\text { ANN, Logistic regression }\end{array}$ & $\begin{array}{l}\text { Overall classification } \\
\text { accuracy: } 92 \% \text { for SVM } \\
\text { and ANN; } 90 \% \text { for } \\
\text { logistic regression; } 88 \% \\
\text { for naïve Bayes; } 83 \% \text { for } \\
\text { classification tree and } \\
\text { kNN (all with 3D texture } \\
\text { features) }\end{array}$ & $\begin{array}{l}\text { Compared with 2D texture } \\
\text { features, 3D texture features } \\
\text { improved diagnostic classification }\end{array}$ \\
\hline Sabin et al.,. ${ }^{15}$ & $\begin{array}{l}38 \text { pts with } \\
\text { histopathologically } \\
\text { proven posterior fossa } \\
\text { ependymoma }\end{array}$ & $\begin{array}{l}\text { Cluster analysis of tumor } \\
\text { location and morphological } \\
\text { variables was performed } \\
\text { to detect tumor } \\
\text { multivariate patterns }\end{array}$ & $\begin{array}{l}\text { Cluster analysis showed } \\
2 \text { tumor groups were } \\
\text { distinguished based on } \\
\text { tumor centroid location. }\end{array}$ & $\begin{array}{l}\text { Such tumor classification was } \\
\text { associated with prognostic and } \\
\text { treatment factors }\end{array}$ \\
\hline Koob et al.,, ${ }^{3}$ & $\begin{array}{l}76 \text { pts (I7 pilocytic } \\
\text { astrocytomas; I6 } \\
\text { embryonal tumors; II } \\
\text { DNET; I0 ependymomas; } \\
\text { other) }\end{array}$ & $\begin{array}{l}\text { Multimodal MRI (TI,T2, } \\
\text { FLAIR, DWI, PWI, MRS) } \\
\text { acquired and parameters } \\
\text { (e.g., rADC) computed; } \\
\text { Multivariate statistical } \\
\text { analysis (using random } \\
\text { forest classifier) performed } \\
\text { to evaluate the diagnostic } \\
\text { accuracy of MR modalities }\end{array}$ & $\begin{array}{l}\text { The highest } \\
\text { diagnostic accuracy } \\
\text { for tumor grading: with } \\
\text { DWI+PWI ( } 73.24 \%) \text {; } \\
\text { and for tumor typing: } \\
\text { with DWI+PWI+MRS } \\
(55.76 \%) \text {. }\end{array}$ & $\begin{array}{l}\text { ADC and } \text { rADC were the best } \\
\text { parameters for tumor grading and } \\
\text { typing; Multimodal MRI can be } \\
\text { accurate in determining pediatric } \\
\text { tumor grades (I and IV) and } \\
\text { types (pilocytic astrocytomas and } \\
\text { embryonal tumors) }\end{array}$ \\
\hline
\end{tabular}

Pts, patients; DWI, diffusion-weighted imaging; MRS, mr spectroscopy; PWI, perfusion-weighted imaging; SVM, support vector machine; ADC, apparent diffusion coefficient; LDA, linear discriminant analysis; KLD, kullback-leibler divergence; $\mathrm{mBm}$, multi-fractional brownian motion; EM, expectation maximization;WMRCTR, west midlands regional childhood tumour registry; PNET, primitive neuroectodermal tumor; PTPSA, piecewise-triangular-prism-surface-area; CCLG, children's cancer and leukemia group; MD, mean diffusivity; FA, fractional anisotropy; PCA, principal component analysis; mRMR, maximum-relevance minimum redundancy; DNET, dysembryoplastic neuroepithelial tumor; kNN, k-nearest neighbour;ANN, artificial neural networks; rADC,ADC tumor-to-normal-brain ratios

Table 2 Summary of multivariate analysis approaches applied to survival/outcome assessment in pediatric brain tumors

\begin{tabular}{|c|c|c|c|c|}
\hline Paper & $\mathbf{N}$ & Methods & Main findings & Other findings \\
\hline Fernandez et al.., ${ }^{16}$ & $\begin{array}{l}80 \text { pts with pilocytic } \\
\text { astrocytomas } \\
\text { ( } 33 \text { cerebellar, } 18 \\
\text { optochiasmatic, } 16 \\
\text { brainstem, } 7 \text { spinal cord, } \\
3 \text { thalamic, } 2 \text { optic nerve, } \\
\text { I hemispheric) }\end{array}$ & $\begin{array}{l}\text { Kaplan-Meier method used } \\
\text { to estimate PFS and OS; } \\
\text { Cox proportional hazards } \\
\text { model used for survival } \\
\text { analysis }\end{array}$ & $\begin{array}{l}\text { 5year PFS rate was } 75 \% \text {, and } \\
\text { the } 5 \text {-year OS rates were } 100 \\
\text { and } 92 \% \text { after total and partial } \\
\text { removal. }\end{array}$ & $\begin{array}{l}\text { Factors such as partial } \\
\text { resection associated with bad } \\
\text { prognosis. }\end{array}$ \\
\hline Bucci et al.., ${ }^{17}$ & $\begin{array}{l}39 \text { pts with } \\
\text { nonbrainstem, malignant } \\
\text { gliomas }\end{array}$ & $\begin{array}{l}\text { Kaplan-Meier method used } \\
\text { to estimate PFS and OS; } \\
\text { Cox proportional hazards } \\
\text { model used for survival } \\
\text { analysis }\end{array}$ & $\begin{array}{l}\text { The median PFS was } \\
12.2 \text { months, and the median } \\
\text { OS was } 21.3 \text { months; } 5 \text {-year } \\
\text { OS and PFS rates of } 35 \% \text { and } \\
26 \%\end{array}$ & $\begin{array}{l}\text { The extent of surgery } \\
\text { and histologic grade were } \\
\text { the strong predictors for } \\
\text { outcomes }\end{array}$ \\
\hline
\end{tabular}


Table Continued..

\begin{tabular}{|c|c|c|c|c|}
\hline Paper & $\mathbf{N}$ & Methods & Main findings & Other findings \\
\hline Dorward et al., ${ }^{18}$ & $\begin{array}{l}40 \text { pts with pilocytic } \\
\text { astrocytoma who } \\
\text { underwent gross-total } \\
\text { resection (GTR) }\end{array}$ & $\begin{array}{l}\text { Kaplan-Meier method } \\
\text { used to estimate survival } \\
\text { time; Cox proportional } \\
\text { hazards regression model } \\
\text { used for recurrence-free } \\
\text { survival analysis }\end{array}$ & $\begin{array}{l}27.5 \%(1 \mathrm{I} / 40) \text { of patients } \\
\text { developed tumor } \\
\text { recurrence after resection, } \\
\text { with a mean time to } \\
\text { recurrence of } 16 \text { months } \\
\text { and a median of } 6.4 \text { months; } \\
\text { Nodular enhancement on } \\
\text { MR imaging at 3-6months was } \\
\text { significantly associated with } \\
\text { recurrence }\end{array}$ & $\begin{array}{l}\text { Postoperative surveillance } \\
\text { MR imaging at } 3-6 \text { months } \\
\text { after resection } \\
\text { predicts tumor recurrence } \\
\text { following GTR. }\end{array}$ \\
\hline $\begin{array}{l}\text { Grech-Sollars et } \\
\text { al., } 19\end{array}$ & $\begin{array}{l}58 \text { pts with embryonal } \\
\text { tumor ( } 40 \text { with } \\
\text { medulloblastoma, } \\
9 \text { with atypical } \\
\text { teratoid/rhabdoid } \\
\text { tumors, and } 9 \text { with } \\
\text { supratentorial primitive } \\
\text { neuroectodermal } \\
\text { tumors) }\end{array}$ & $\begin{array}{l}\text { ACTC (a measure of the } \\
\text { gradient change of ADC } \\
\text { from the peri-tumoral } \\
\text { edema into the tumor } \\
\text { core) extracted from } \\
\text { DWI images; Multivariate } \\
\text { survival analysis used } \\
\text { Cox proportional hazard } \\
\text { regression }\end{array}$ & $\begin{array}{l}\text { More negative ATCT values } \\
\text { are significantly associated } \\
\text { with a poorer survival } \\
\text { (regardless of tumor type, } \\
\text { extent of resection, age } \\
<3 y \text { ears at diagnosis, and } \\
\text { metastasis at presentation) }\end{array}$ & $\begin{array}{l}\text { There is a significant difference } \\
\text { for survival data regarding the } \\
\text { change in ADC from edema } \\
\text { into the tumor volume. }\end{array}$ \\
\hline Youland et al., ${ }^{20}$ & $\begin{array}{l}35 \text { I pts with low-grade } \\
\text { gliomas ( } 68 \text { pilocytic, } \\
88 \text { astrocytoma; } 47 \\
\text { oligodendroglioma; } \\
24 \text { subependymal } \\
\text { giant cell; } 20 \text { mixed } \\
\text { oligoastrocytoma; } 4 \\
\text { other/unknown) }\end{array}$ & $\begin{array}{l}\text { Kaplan-Meier method used } \\
\text { to estimate PFS and OS; } \\
\text { Cox proportional hazards } \\
\text { model used for survival } \\
\text { analysis }\end{array}$ & $\begin{array}{l}\text { I0-year PFS was } 62 \% \text { and OS } \\
\text { was } 90 \% \text {; Improved PFS was } \\
\text { associated with GTR and } \\
\text { postoperative RT; higher OS } \\
\text { was associated with GTR and } \\
\text { pilocytic histology }\end{array}$ & $\begin{array}{l}\text { GTR was associated with } \\
\text { improved OS and PFS; RT } \\
\text { associated with improved PFS }\end{array}$ \\
\hline Sun et al., ${ }^{21}$ & $\begin{array}{l}33 \text { pts with brainstem } \\
\text { gliomas ( } 8 \text { astrocytoma, } \\
3 \text { oligodendroglioma, } \\
2 \text { oligoastrocytoma, I } \\
\text { glioblastoma, other) }\end{array}$ & $\begin{array}{l}\text { Kaplan-Meier method } \\
\text { used to estimate survival } \\
\text { time; Cox proportional } \\
\text { hazards regression model } \\
\text { used for survival analysis }\end{array}$ & $\begin{array}{l}\text { Overall median survival } \\
\text { I Imonths with I-year survival } \\
\text { rate } 43.6 \% \text {; Multivariate } \\
\text { analysis showed that } \\
\text { diagnostic latency ( }<2 \text { months) } \\
\text { and tumor focality were } \\
\text { associated with longer survival }\end{array}$ & $\begin{array}{l}\text { Diffuse pattern of tumor was } \\
\text { markedly associated with a } \\
\text { shorter survival }\end{array}$ \\
\hline Sun et al., ${ }^{22}$ & $\begin{array}{l}102 \text { pts with choroid } \\
\text { plexus carcinoma }\end{array}$ & $\begin{array}{l}\text { Kaplan-Meier and } \\
\text { multivariate Cox } \\
\text { regression survival analyses } \\
\text { performed to determine } \\
\text { the effect of GTR }\end{array}$ & $\begin{array}{l}\text { Multivariate analysis showed } \\
\text { that GTR increased OS }\end{array}$ & $\begin{array}{l}\text { GTR improved PFS on } \\
\text { Kaplan-Meier analysis, but } \\
\text { not significant in multivariate } \\
\text { analysis }\end{array}$ \\
\hline Felix et al., ${ }^{23}$ & $\begin{array}{l}19 \text { pts with diffuse } \\
\text { intrinsic pontine gliomas }\end{array}$ & $\begin{array}{l}\text { Kaplan-Meier method } \\
\text { used to estimate survival } \\
\text { time; Cox proportional } \\
\text { hazards regression model } \\
\text { used for survival analysis }\end{array}$ & $\begin{array}{l}\text { Median OS of patients in } \\
\text { treated vs. non-treated group: } \\
\text { I } 3.4 \text { vs. } 7.8 \text { months. Median } \\
\text { event-free survival of patients } \\
\text { in the treated vs. non-treated } \\
\text { group: } 9.5 \text { vs. } 6.5 \text { months }\end{array}$ & $\begin{array}{l}\text { Significant longer survival in } \\
\text { the treated group than non- } \\
\text { treated group }\end{array}$ \\
\hline Jansen et al., ${ }^{24}$ & $\begin{array}{l}316 \text { pts with diffuse } \\
\text { intrinsic pontine glioma }\end{array}$ & $\begin{array}{l}\text { Cox proportional } \\
\text { hazards with backward } \\
\text { regression was used to } \\
\text { select prognostic variables } \\
\text { for survival prediction; } \\
\text { bootstrapping was used to } \\
\text { validate the model }\end{array}$ & $\begin{array}{l}\text { Median overall survival was } \\
\text { IOmonths; } A \cup C=0.68\end{array}$ & $\begin{array}{l}\text { Positive predictors:Age } \\
\leq 3 y e a r s, \text { longer symptom } \\
\text { duration at diagnosis, and } \\
\text { use of oral and intravenous } \\
\text { chemotherapy; Negative } \\
\text { predictor: ring enhancement } \\
\text { on MRI }\end{array}$ \\
\hline
\end{tabular}


Table Continued.

\begin{tabular}{|c|c|c|c|c|}
\hline Felicetti et al., ${ }^{25}$ & $\begin{array}{l}90 \text { pts with tumor ( } 44 \\
\text { non-Hodgkin lymphoma; } \\
19 \text { medullo-blastoma; } \\
7 \text { ependymoma; } \\
6 \text { astrocytoma; } 4 \\
\text { germinoma; } 10 \text { others) } \\
\text { who underwent } \\
\text { radiotherapy }\end{array}$ & $\begin{array}{l}\text { Cox multivariable analysis } \\
\text { was performed to identify } \\
\text { potential risk factors }\end{array}$ & $\begin{array}{l}\text { The occurrence of } \\
\text { meningioma was associated } \\
\text { with the development of } \\
\text { other second neoplasms }\end{array}$ & $\begin{array}{l}\text { Age, sex, or CRT dose had no } \\
\text { influence on the occurrence of } \\
\text { meningioma }\end{array}$ \\
\hline Morana et al., ${ }^{26}$ & $\begin{array}{l}2 \text { I pts with glioma ( } 9 \\
\text { diffuse astrocytoma; } 5 \\
\text { anaplastic astrocytoma; } \\
5 \text { glioblastoma; I } \\
\text { glioneuronal tumor; } \\
\text { other) }\end{array}$ & $\begin{array}{l}\text { (I8)F-DOPA PET and } \\
\text { MRS data were compared } \\
\text { and correlated; Cox } \\
\text { multivariable analysis was } \\
\text { performed for survival } \\
\text { analysis }\end{array}$ & $\begin{array}{l}\text { Diagnostic accuracy: (I8) } \\
\text { F-DOPA PET: 78\%; MRS: } \\
\text { 93\%; (I8)F-DOPA uptake } \\
\text { correlated with PFS and OS }\end{array}$ & $\begin{array}{l}\text { Significant differences of ( } 18) \\
\text { F-DOPA uptake and MRS } \\
\text { ratios were found between } \\
\text { low-grade and high-grade } \\
\text { gliomas }\end{array}$ \\
\hline Gunther et al.,. ${ }^{27}$ & 72 pts with ependymoma & $\begin{array}{l}\text { Multivariate analysis was } \\
\text { used to determine RT } \\
\text { effect; Cox multivariable } \\
\text { analysis was performed for } \\
\text { survival analysis }\end{array}$ & $\begin{array}{l}\text { Proton beam RT (compared } \\
\text { to intensity modulated RT) } \\
\text { was associated with more } \\
\text { frequent imaging changes }\end{array}$ & $\begin{array}{l}\text { Postradiation MRI changes are } \\
\text { more common with proton } \\
\text { beam RT and in patients less } \\
\text { than 3years of age at diagnosis } \\
\text { and treatment }\end{array}$ \\
\hline Li et al., ${ }^{28}$ & 79 pts with meningiomas & $\begin{array}{l}\text { Univariate and multivariate } \\
\text { analyses used to examine } \\
\text { the association between } \\
\text { high-grade meningiomas } \\
\text { and imaging features }\end{array}$ & $\begin{array}{l}\text { An unclear tumor- } \\
\text { brain interface, lateral location, } \\
\text { and narrow base were } \\
\text { predictive factors for high- } \\
\text { grade meningiomas }\end{array}$ & $\begin{array}{l}\text { Pediatric meningiomas have } \\
\text { specific imaging features on } \\
\text { MRI indicating their malignancy. }\end{array}$ \\
\hline Steinbok et al., ${ }^{29}$ & $\begin{array}{l}72 \text { pts with thalamic } \\
\text { tumors }\end{array}$ & $\begin{array}{l}\text { Kaplan-Meier method } \\
\text { used to estimate survival } \\
\text { time; Cox proportional } \\
\text { hazards regression model } \\
\text { used for survival analysis }\end{array}$ & $\begin{array}{l}5 \text {-year overall survival was } \\
6 I \pm I 3 \% \text { for unithalamic } \\
\text { tumors compared to } 37 \pm 32 \% \\
\text { for bithalamic tumors; } \\
\text { Highgrade tumors had a } \\
\text { much lower } 5 \text {-year OS }(7 \pm 13 \\
\%) \text { than low-grade tumors } \\
(84 \pm 17 \%) \text {; }\end{array}$ & $\begin{array}{l}\text { Unilateral tumors were mainly } \\
\text { low grade vs. bithalamic } \\
\text { tumors (high-grade). } \\
\text { Multivariate analysis indicated } \\
\text { tumor grade as the only } \\
\text { significant prognostic factor } \\
\text { for unithalamic tumors }\end{array}$ \\
\hline
\end{tabular}

Pts, patients; AUC, area under the ROC (Receiver Operating Characteristic) curve; ATCT, apparent transient coefficient in tumor; OS, overall survival; PFS, progression-free survival; GTR, gross total resection; RT, radiation therapy; DNT, dysembryoplastic neuroepithelial tumor

\section{Multivariate analysis in pediatric brain tumor classifi- cation or segmentation}

In the early years, multivariate methods such as LDA and belief networks were used in tumor classification (Table 1). Schneider et al. computed imaging parameters (ADC and metabolite ratio features from MRI, DWI and MRS), and used LDA as classifier to discriminate between four tumor groups (medulloblastoma, infiltrating glioma, ependymoma, and pilocytic astrocytoma). ${ }^{4}$ They found that combined $\mathrm{ADC}$ and metabolite ratio features (using water as an internal standard) could discriminate between these tumor groups. ${ }^{4}$ Reynolds et al. used tumor location information obtained from MRI to construct Bayesian belief networks, incorporated MRS information into the classifiers, and found that inclusion of a priori knowledge improved classification accuracy. ${ }^{5}$ Later, Davies et al. extracted multiple metabolite profiles from (short-TE PRESS) MRS, classified tumors (e.g., glial-cell vs. non-glial-cell tumors) with LDA and achieved high classification accuracies $(>90 \%) .^{7}$ Further, in a multi-national study $(\mathrm{n}=78)$, Vicente et al. computed metabolite concentrations from (short-TE PRESS) MRS data, classified tumors (e.g., medulloblastomas vs. ependymomas vs. pilocytic astrocytomas) with LDA, and achieved high classification accuracies $(98 \% \sim 100 \%){ }^{11}$

Tumor segmentation allows quantitative (e.g., tumor volume) analysis, and automatic tumor segmentation was achieved by applying graph cut method to tumor image and using probabilistic boosting trees as classifier. ${ }^{6}$ In addition, Ahmed et al. $^{8}$ and Iftekharuddin et al., ${ }^{9}$ used shape, intensity and texture features extracted from MRI images for tumor segmentation and classification (sensitivity $100 \%$, specificity $75 \%){ }^{9}$ For special brain tumors such as optic pathway gliomas, automatic tumor segmentation was achieved by using prior tumor location tissue characteristics and intensity information, and by classifying tumor into internal components. ${ }^{10}$

In recentyears, multivariate analysis methods such as SVM, cluster analysis and random forest have been applied to tumor classification (typing) and tumor grading. Using SVM classifier, Rodriguez Gutierrez et al. demonstrated that ADC histogram features generated high classification accuracy (95.8\% of medulloblastomas, $96.9 \%$ of pilocytic astrocytomas, and $94.3 \%$ of ependymomas); and ADC textural features produced high tumor-subtype classification accuracy ( $89 \%$ of medulloblastoma subtypes). ${ }^{12}$ With a large sample $(n=74)$, Tantisatirapong et al. investigated two feature selection approaches (principal component analysis PCA, and the combination of maxrelevance and min-redundancy (mRMR) and feedforward selection), and tumor classification with SVM classifier based on texture features (from MRI, DWI and DTI) yielded varied classification performance. ${ }^{13}$ In addition, Fetit et al. examined the classification 
performance of 6 classifiers with 2D and 3D texture features extracted MRI (T1, T2) and found that SVM and ANN had the highest overall classification accuracy (92\%), and 3D texture features improved classification performance. ${ }^{14}$ In patients with pediatric posterior fossa ependymoma, tumor classification with cluster analysis revealed that tumor subgroups could be distinguished based on tumor centroid location, which was associated with prognostic and treatment factors. ${ }^{15}$ Further, in a recent study $(n=76)$, Koob et al. classified tumors with random forest classifier, and found diagnostic accuracy $73.24 \%$ with DWI+PWI for tumor grading, and $55.76 \%$ with DWI+PWI+MRS for tumor typing. ${ }^{3}$ They also found that the best parameters for tumor grading and typing were ADC and rADC (ADC tumor-to-brain ratios), and multimodal MRI (MRI, DWI, PWI, MRS) could determine pediatric tumor grades (I, IV) and types (pilocytic astrocytomas and embryonal tumors). ${ }^{3}$

However, there are large variations in the tumor classification results among the studies (even in similar patient samples), e.g., ${ }^{12}$ vs. ${ }^{13} ;{ }^{3}$ vs. ${ }^{11} ;{ }^{3}$ vs. ${ }^{12}$

\section{Multivariate analysis in survival/outcome assessment in pediatric brain tumors}

The majority of the studies listed in Table 2 used Kaplan-Meier method to estimate progression-free survival (PFS) and overall survival (OS), and applied multivariate Cox proportional hazards model to identification of prognostic factors. In patients with pilocytic astrocytomas $(\mathrm{n}=80)$, 5year PFS rate was $75 \%$, and the 5 year OS rates were $92-100 \%{ }^{16}$ In patients with low-grade gliomas $(\mathrm{n}=351)$, 10year PFS was $62 \%$ and OS was $90 \% .{ }^{20}$ However, in patients with malignant non-brainstem gliomas $(n=39)$, 5year OS and PFS rates were $35 \%$ and $26 \%$ respectively, and the median PFS and OS were 12.2 months and 21.3 months respectively. ${ }^{17}$ In addition, in patients with brainstem gliomas $(\mathrm{n}=33)$, overall median survival was 11 months with 1year survival rate $43.6 \% .{ }^{21}$ Further, diffuse intrinsic pontine glioma has the worst prognosis. The median overall survival was 10 months in patients with diffuse intrinsic pontine glioma $(n=316)$ and in this large patient sample, the area under the ROC (Receiver Operating Characteristic) curve (AUC) for the final multivariate survival model was $0.68 .^{24}$

A number of prognostic factors such as tumor characteristics (type, grade, location, etc.), imaging features and treatment are associated with patients' survival. ${ }^{17,19,20,23-26}$ The AUC (0.68) of the final multivariate survival model for a large sample of patient with diffuse intrinsic pontine glioma $(n>300)^{24}$ indicates that the survival estimate and prognostic factor identification made by the multivariate survival model are far from perfect, and error may exist in the survival results.

\section{Discussion}

Brain tumors in children are heterogeneous, and have imaging and histological features that are quite different from adults. Posterior fossa tumors are the most studied pediatric brain tumors. Multivariate approaches are powerful analytic tools suitable for high dimensional problems, and have been increasingly used in pediatric brain tumor classification (or segmentation) and patient survival (outcome) assessment.

\section{Pediatric brain tumor classification/segmentation with multivariate analysis}

The large variations in the classification results among the imaging studies (even in similar samples) may be explained by viewing the process of brain tumor classification as an image processing chain, and the final classification result (classification accuracy) as the imaging diagnostic accuracy. A number of factors along the tumor classification processing chain have impacts on the final result.

First, image selection for analysis: The most commonly used imaging data for tumor classification is MRI (T1, T2, FLAIR); DWI and MRS are often used; and PWI is sometimes used. Although the diagnostic values of advanced imaging modalities such as PWI and the optimal MR imaging protocol for tumor classification are still to be determined, studies have shown that metabolite ratios (from MRS) or ADC features (from DWI) alone could not discriminate the tumor groups, but combined ADC and metabolite ratio features could $;{ }^{4}$ further, the combination of DWI, MRS and PWI was good for tumor classification (accuracy $55.76 \%$ ), and the combination of DWI and PWI was good for tumor grade evaluation (accuracy 73.24\%). ${ }^{3}$ More studies are needed to examine the diagnostic values of advanced MRI imaging modalities for each tumor type and grade, and explore the optimal MR imaging protocol for classification of pediatric brain tumors.

Second, feature extraction: Some imaging features such as shape, histogram, tumor location, $\mathrm{ADC}$, metabolite concentration ratio and CBV (cerebral blood volume) can be obtained by straightforward standard computation. However, some imaging features such as texture features are not straightforward nor standard. They can be $2 \mathrm{D}$ or $3 \mathrm{D}$, based on grey-level co-occurrence matrix or grey-level run-length matrix,${ }^{14}$ or fractal dimension or multi-fractional Brownian motion. ${ }^{8,9}$ Study has shown that computed with 2D texture features, 3D texture features improved tumor classification performance. ${ }^{14}$ In addition, it has been shown that multi-fractional Brownian motion-based texture features produced high tumor segmentation accuracy for T1 and FLAIR images and for T1-T2-FLAIR fused images (100\%), and intensity features best for T2 images. ${ }^{8}$ Therefore, classification performance can be improved by standardizing (e.g., 3D texture features) and optimizing feature extraction.

Third, feature selection: Studies have investigated various feature selection approaches including: PCA, boosting, KLD (Kullback-Leibler divergence) ${ }^{8}$ entropy (entropy-MDL discretization) $;{ }^{14}$ mRMR (maximum-relevance minimum redundancy) and feed forward methods,${ }^{8}$ and expectation maximization for feature fusion. ${ }^{8}$ Since feature selection methods determine which features to be used for classification analysis, the quality of these methods directly affects classification result. Further research is needed to optimize and standardize feature selection to improve tumor classification.

Fourth, classification: A variety of multivariate classifiers such as LDA, SVM, ANN, cluster analysis and random forest have been applied to tumor classification. The classification performances of 6 classifiers (naïve Bayes, k-nearest neighbor $(\mathrm{kNN})$, classification tree, SVM, ANN, logistic regression) have been examined in a recent study which reported the tumor classification results, i.e., the overall classification accuracy of these classifiers (using 3D texture features): $92 \%$ for SVM and ANN; $90 \%$ for logistic regression; $88 \%$ for naïve Bayes; $83 \%$ for classification tree and $\mathrm{kNN} .{ }^{14}$ The large differences in classification performances (e.g., $83 \%$ vs. $92 \%$ ) indicates the importance of selecting the best classifier for the data (for patients with specific tumor types and grades), standardizing classifier for each tumor type and grade, and optimizing the classifier for the best classification performance.

Taken together, variations in each step along the imaging processing 
chain of tumor classification could accumulate and cause the large variations in final classification results among the studies. Therefore, optimizing and standardizing the imaging processing chain of tumor classification may reduce the large variations of classification results among the studies, improve classification performance, and enhance the diagnostic values of MRI imaging modalities.

\section{Survival/outcome assessment with multivariate analy- sis in pediatric brain tumors}

Compared with tumor classification studies, the survival assessmen studies have larger sample sizes ( 2 studies have sample sizes $>300$ ) and there are less variations in the survival results among the studies. However, as indicated in the AUC (0.68) of the final multivariate survival model, ${ }^{24}$ the error rate in the multivariate survival analysis is un-neglectable. The process of survival assessment in pediatric brain tumors can also be viewed as a data processing chain, and several factors along the chain have impacts on the final results.

First, data selection for analysis: A wide range of data can be used for patients' survival assessment (patient's age, gender, symptoms, tumor characteristics, diagnostic latency, treatments, etc.). Some data needs to be extracted or computed from raw data. For example, imaging features such as ACTC (Apparent transient coefficient in tumor, a measure of the gradient change of ADC from the peri-tumoral edema into the tumor core) extracted from DWI are associated with survival. ${ }^{19}$ Since each data contributes to the final survival results differently (i.e., each has different weight) and some data are of low quality, data needs to be screened and selected (e.g., according to their weights) for survival analysis. Different data selection affects survival estimate. Thus, optimizing and standardizing data selection may reduce errors and improve survival analysis.

Second, statistical analysis: Survival assessment is a standard approach:

a) Use Kaplan-Meier method to estimate progression-free survival (PFS) and overall survival (OS), and compute differences in OS between different groups (tumor groups or grades) with univariate analysis through Kaplan-Meier survival plots;

b) Use multivariate Cox proportional hazards model to evaluate the significance of associated data variables (prognostic factors that are associated with survival) indicated by the univariate analysis.

However, the methods that select data variables or prognostic factors may vary among studies (either from univariate analysis to multivariate analysis, or in refining the multivariate analysis model). Bootstrapping is needed to validate the final multivariate model so that the results of survival analysis may be more accurate and the final model may be generalizable. ${ }^{24}$ Taken together, optimizing and standardizing the data processing chain in survival assessment may reduce errors, improve survival estimation, and enhance the identification of prognostic factors in survival analysis.

A method comparison between multivariate analyses in tumor classification and survival assessment is presented in Table 3. Compared with relatively complex imaging data and their processing in tumor classification, the relatively simple data and their processing in survival assessment make it possible to perform multivariate survival analysis on large samples (e.g., $\mathrm{n}>300$ ), which makes the results of survival analysis (based on large samples) more stable and reliable.

Table 3 Method comparison of multivariate analyses in tumor classification vs. survival assessment

\begin{tabular}{|c|c|c|}
\hline & Tumor classification & Survival assessment \\
\hline Sample sizes* & $>=6$ and $<=78$ & $>=19$ and $<=351$ \\
\hline Data for analysis & $\begin{array}{l}\text { MRI (TI,T2, FLAIR) and/or DWI, MRS, } \\
\text { PWI, etc. }\end{array}$ & $\begin{array}{l}\text { Age, gender, symptom(s) duration, tumor characteristics (location, } \\
\text { focality, type, grade, size, shape, imaging intensity, whether ring } \\
\text { enhancement on MRI, whether unclear tumor-brain interface, } \\
\text { whether development of other second neoplasms, whether } \\
\text { abnormality on other imaging such as DWI, MRS and PET, } \\
\text { recurrence, etc.), diagnostic latency, treatment (whether use of } \\
\text { chemotherapy, whether use of radiotherapy, dose, whether surgical } \\
\text { treatment, extent of resection, histology, etc.) }\end{array}$ \\
\hline
\end{tabular}

Features with standard computation: shape, intensity, histogram, tumor location, ADC for DWI, metabolite concentrations or ratios for MRS, CBV (cerebral blood volume) for PWI, etc.; Features with varied computation: texture features (2D vs. 3D, grey-level co-occurrence matrix-based vs. fractal dimension and multi-fractional Brownian motion-based)

Use feature selection (or reduction) method: PCA, boosting, KLD (Kullback-Leibler divergence), entropy (entropy-MDL discretization); or mRMR (maximum-relevance minimum redundancy) and feedforward methods; expectation maximization for feature fusion, etc.
Extract data such as imaging features (if necessary), e.g., ACTC (a measure of the gradient change of ADC from the peri-tumoral edema into the tumor core) (Grech-Sollars et al. 2012) ${ }^{19}$

Use Kaplan-Meier method to estimate progression-free survival (PFS) and overall survival (OS); Compute differences in OS between different groups (tumor groups or grades) with univariate analysis through Kaplan-Meier survival plots; Select significant prognostic factors in univariate analysis 
Table Continued.

\begin{tabular}{lll}
\hline & Tumor classification & Survival assessment \\
\hline Sample sizes* & $>=\mathbf{6}$ and $<=\mathbf{7 8}$ & $>=19$ and $<=\mathbf{3 5 I}$ \\
\hline $\begin{array}{l}\text { Classification/ Multivariate } \\
\text { analysis }\end{array}$ & $\begin{array}{l}\text { Use classification method: LDA, SVM, } \\
\text { ANN, cluster analysis, random forest, } \\
\text { etc. }\end{array}$ & $\begin{array}{l}\text { Use multivariate Cox proportional hazards model to evaluate the } \\
\text { significance of associated data variables (prognostic factors that are } \\
\text { associated with survival) indicated by the univariate analysis }\end{array}$ \\
Model validation & Cross-validation, etc. & Bootstrapping, etc.
\end{tabular}

*Sample sizes, based on the literature reviewed;ATCT, apparent transient coefficient in tumor; OS, overall survival; PFS, progression-free survival

\section{Conclusion}

In summary, this paper reviewed the studies that applied multivariate analysis to tumor classification (segmentation) and survival (outcome) assessment in pediatric brain tumors. The large variations in the classification results among the tumor classification studies (even in similar patient populations) may be reduced by optimizing and standardizing image processing chains of tumor classification. Similarly, optimizing and standardizing data processing chains of survival assessment may improve survival analysis and reduce errors in survival estimates and identification of prognostic factors.

As multivariate analytic approaches, data processing technologies and imaging techniques advance in the Big Data era of the $21^{\text {st }}$ century, it is anticipated that the obstacles in the complex imaging data processing in tumor classification will be overcome and complex data processing will be revolutionized. This will make accurate automatic tumor classification/segmentation (for each tumor type and grade) possible to early detect and treat tumors, guide treatment planning, monitor tumor progression and treatment effects, together with advanced survival assessment to guide life-saving rescue and recovery planning, revolutionize patient care, and truly benefit children with brain tumors.

\section{Acknowledgements}

None.

\section{Conflict of interest}

Author declares that there is no conflict of interest.

\section{References}

1. http://www.cancerresearchuk.org/cancer-info/cancerstats/childhoodcancer/incidence/childhood-cancer-incidence-statistics

2. Levman J, Takahashi E. Pre-Adult MRI of Brain Cancer and Neurological Injury: Multivariate Analyses. Front Pediatr. 2016;4:65.

3. Koob M, Girard N, Ghattas B, et al. The diagnostic accuracy of multiparametric MRI to determine pediatric brain tumor grades and types. $J$ Neurooncol. 2016;127(2):345-353.

4. Schneider JF, Confort-Gouny S, Viola A, et al. Multiparametric differentiation of posterior fossa tumors in children using diffusion-weighted imaging and short echo-time 1H-MR spectroscopy. J Magn Reson Imaging. 2007;26(6):1390-1398.

5. Reynolds GM, Peet AC, Arvanitis TN. Generating prior probabilities for classifiers of brain tumours using belief networks. BMC Med Inform Decis Mak. 2007;7:27.
6. Wels M, Carneiro G, Aplas A, et al. A discriminative model-constrained graph cuts approach to fully automated pediatric brain tumor segmentation in 3-D MRI. Med Image Comput Comput Assist Interv. 2008;11(Pt 1):67-75.

7. Davies NP, Wilson M, Harris LM, et al. Identification and characterisation of childhood cerebellar tumours by in vivo proton MRS. NMR Biomed. 2008;21(8):908-918.

8. Ahmed S, Iftekharuddin KM, Vossough A. Efficacy of texture, shape, and intensity feature fusion for posterior-fossa tumor segmentation in MRI. IEEE Trans Inf Technol Biomed. 2011;15(2):206-213.

9. Iftekharuddin KM, Ahmed S, Hossen J. Multiresolution texture models for brain tumor segmentation in MRI. Conf Proc IEEE Eng Med Biol Soc. 2011;2011:6985-6988.

10. Weizman L, Ben Sira L, Joskowicz L, et al. Automatic segmentation, internal classification, and follow-up of optic pathway gliomas in MRI. Med Image Anal. 2012;16(1):177-188.

11. Vicente J, Fuster-Garcia E, Tortajada S, et al. Accurate classification of childhood brain tumours by in vivo (1)H MRS-a multi-centre study. Eur J Cancer. 2013;49(3):658-667.

12. Rodriguez Gutierrez D, Awwad A, Meijer L, et al. Metrics and textural features of MRI diffusion to improve classification of pediatric posterior fossa tumors. AJNR Am J Neuroradiol. 2014;35(5):1009-1015.

13. Tantisatirapong S, Davies NP, Rodriguez D, et al. Magnetic resonance texture analysis: Optimal feature selection in classifying child brain tumors. XIII Mediterranean Conference on Medical and Biological Engineering and Computation. 2014;41:309-312.

14. Fetit AE, Novak J, Peet AC, et al. Three-dimensional textural features of conventional MRI improve diagnostic classification of childhood brain tumours. NMR Biomed. 2015;28(9):1174-1184.

15. Sabin ND, Merchant TE, Li X, et al. Quantitative imaging analysis of posterior fossa ependymoma location in children. Childs Nerv Syst. 2016;32(8):1441-1447.

16. Fernandez C, Figarella-Branger D, Girard N, et al. Pilocytic astrocytomas in children: prognostic factors--a retrospective study of 80 cases. Neurosurgery. 2003;53(3):544-553.

17. Bucci MK, Maity A, Janss AJ, et al. Near complete surgical resection predicts a favorable outcome in pediatric patients with nonbrainstem, malignant gliomas: results from a single center in the magnetic resonance imaging era. Cancer. 2004;101(4):817-824.

18. Dorward IG, Luo J, Perry A, et al. Postoperative imaging surveillance in pediatric pilocytic astrocytomas. $J$ Neurosurg Pediatr. 2010;6(4):346-352.

19. Grech-Sollars M, Saunders DE, Phipps KP, et al. Survival analysis for apparent diffusion coefficient measures in children with embryonal brain tumours. Neuro Oncol. 2012;14(10):1285-1293. 
20. Youland RS, Khwaja SS, Schomas DA, et al. Prognostic factors and survival patterns in pediatric low-grade gliomas over 4 decades. J Pediatr Hematol Oncol. 2013;35(3):197-205.

21. Sun T, Wan W, Wu Z, et al. Clinical outcomes and natural history of pediatric brainstem tumors: with 33 cases follow-ups. Neurosurg Rev. 2013;36(2):311-319.

22. Sun MZ, Ivan ME, Clark AJ, et al. Gross total resection improves overall survival in children with choroid plexus carcinoma. $J$ Neurooncol. 2014;116(1):179-185.

23. Felix FH, de Araujo OL, da Trindade KM, et al. Retrospective evaluation of the outcomes of children with diffuse intrinsic pontine glioma treated with radiochemotherapy and valproic acid in a single center. $J$ Neurooncol. 2014;116(2):261-266.

24. Jansen MH, Veldhuijzen van Zanten SE, SanchezAliaga E, et al. Survival prediction model of children with diffuse intrinsic pontine glioma based on clinical and radiological criteria. Neuro Oncol. 2015;17(1):160-166.

25. Felicetti F, Fortunati N, Garbossa D, et al. Meningiomas after cranial radiotherapy for childhood cancer: a single institution experience. $J$ Cancer Res Clin Oncol. 2015;141(7):1277-1282.
26. Morana G, Piccardo A, Puntoni M, et al. Diagnostic and prognostic value of 18F-DOPA PET and 1H-MR spectroscopy in pediatricsupratentorial infiltrative gliomas: a comparative study. Neuro Oncol. 2015;17(12):1637-1647.

27. Gunther JR, Sato M, Chintagumpala M, et al. Imaging Changes in Pediatric Intracranial Ependymoma Patients Treated With Proton Beam Radiation Therapy Compared to Intensity Modulated Radiation Therapy. Int J Radiat Oncol Biol Phys. 2015;93(1):54-63.

28. Li H, Zhao M, Jiao Y, et al. Prediction of High-Grade Pediatric Meningiomas: Magnetic Resonance Imaging Features Based on T1-Weighted, T2-Weighted, and Contrast-Enhanced T1-Weighted Images. World Neurosurg. 2016;91:89-95.

29. Steinbok P, Gopalakrishnan CV, Hengel AR, et al. Pediatric thalamic tumors in the MRI era: a Canadian perspective. Childs Nerv Syst. 2016;32(2):269-280. 